\title{
A New Approach of Feature Extraction using Genetic Algorithm and SIFT
}

\author{
Kapil Kumar Pachouri \\ Department of Computer Science \& Engineering \\ Oriental Institute of Science \& Technology \\ Bhopal, India
}

\author{
Atul Barve \\ Department of Computer Science \& Engineering \\ Oriental Institute of Science \& Technology \\ Bhopal, India
}

\begin{abstract}
Feature Extraction is a process of extracting the point from the image such that the image can be compared and recognized easily and quickly. Since various feature extraction techniques are implemented so that it can be used for variety of applications such as face recognition. The existing technique implemented for the extraction of features such as SIFT, SURF are efficient in terms of accuracy of number of features extraction but can't extracted all features from different types of images. Hence an efficient technique is implemented here for the efficient features extracted from images using combinatorial method of optimizing SIFT features extraction technique so that more number of features can be extracted.
\end{abstract}

\section{Keywords}

SIFT, Genetic Algorithm, SURF, HFE, SVM.

\section{INTRODUCTION}

Facial feature extraction is a vital stage in the face detection and facial expression recognition structures. The extracted features contain significant information of the face that designates the face performance. To improve a better facial expression recognition arrangement, a noble feature extraction process is essential so that the system can effectively distinguish dissimilar facial expressions. In this study area, feature extraction is the most challenging and interesting task.The world we live in is highly go-ahead and understanding of dynamic processes is essential in all the fields that have to deal with moving objects or occurrences. It can be strongly maintenance by visual explorative techniques and collaborative tools. The visual analytics which is essentially an emerging discipline integrating user interfaces with multidimensional and multivariate visualization tools such as maps, diagrams and graphs, etc.

Different kinds of self-motivated objects and events are comprehensively discussed with an emphasis on the specification of their semantic and geometrical attributes. Furthermore, people interact on certain places at or during certain times with each other and with our environment. This spatio-temporal activities also lead to movements and changes, such as shifting of boundaries (e.g. political or traditional ones). Accepting of undertakings is an essential conceptual process for the informed decisions in the modern society. The first step for a computer program in semantic understanding, nevertheless, is to abstract well-organized and actual visual features and build models from them rather than human background knowledge. So we can see that how to extract image low-level visual features and what kind of features will be extracted play a crucial role in various tasks of image processing. As we known, the most common visual features include color, texture and shape, etc. [1-3], and most image annotation and retrieval systems have been constructed based on these features. However, their performance is heavily dependent on the use of image features. Texture is a very useful characterization for a wide range of image.

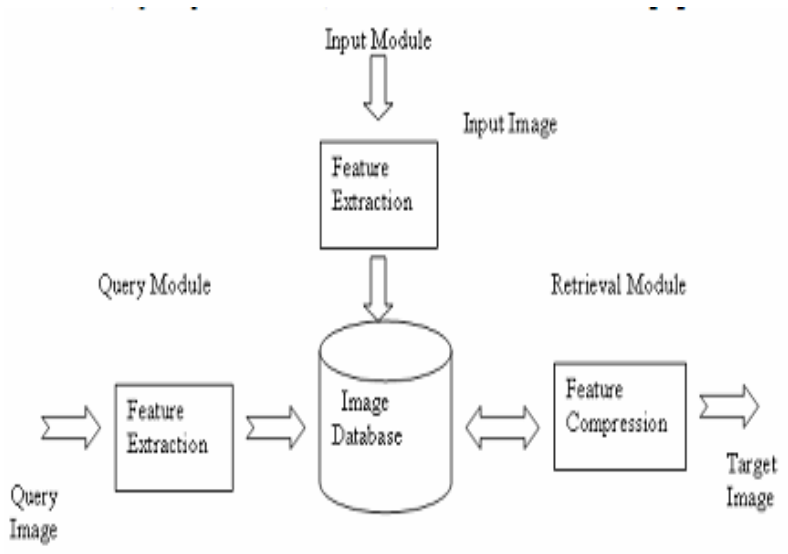

Figure.1. Block diagram of image retrieval system

The general image retrieval system is shown in Figure 1. It consists of three main modules such as input module, query module, and retrieval module [4]. In the input module, the feature vector is extracted from input image. It is then stored along with its input image in the image database. On the other hand, when a query image enters the query module, it extracts the feature vector of the query image.

\section{LITERATURE SURVEY}

In this paper [5] author has make use of a quantitative, strong calculation to estimate the spatial exposure of feature points in an image, intelligent to establish whether points are comprehensive at multiple scales. When matching images for relevance's such as mosaicking and homography evaluation, the distribution of features across the overlap region affects the accuracy of the result show that SFOP commences extensively less aggregation than the other detectors tested and it is measured by Ripley's K-function, to evaluate whether feature matches are clustered simultaneously or increase approximately the have common characteristics region. Based on this determine, an appraisal of a range of up to date feature detectors and then carried out using analysis of variance and a large image database was executed; the estimate method considered the imagery and the detector as the two self-determining variables have an effect on coverage, and consequence was evaluated using ANOVA. The results revealed that there is indeed statistical significance between the performances of detectors. When the detectors are rankordered by this act calculate, the order is generally comparable to those get hold of by other means, put it to some 
bodying that the arranging reveals authentic performance differences. SFOP was found to be better-quality to other detectors, while there are also some detectors whose performance differences were not statistically important. These findings are generally dependable with those get hold of by other investigators using unusual come within reach of, increasing our self-assurance that these concert differences are authentic. Researches were also try to get completed on stitching have common characteristics regions into landscapes, substantiating that enhanced reporting give ways a better quality consequence.

In this paper [6], author has tried to find on the MediaMill challenge problem with a new method that is HFE method using the walk-based graph kernel. A set of features such as size, color, and texture are associated with each segment. We consider a method using support vector machines (SVMs) with walk-based graph kernels for high-level feature extraction from images.

Our aim is to extend a method of high-level feature extraction (HFE) from images, e.g., detecting whether an image is a background or contains an object such as a car or a dog. If a list of conceptions such as "is a landscape" or "contains a dog" is given, this task can also be considered as a set of take charge of binary classification tasks, where each image must be assigned a set of binary labels to indicate whether or not it belongs to each concept class. Unlike more precise undertakings such as face or character recognition, the importance in HFE is on acquiring nonspecific and multipurpose automatic tools that can "learn" any conception from a set of examples be in the right placing to the concept class. So the author gets acquired a relative increase of $58 \%$ measure up to with the baseline concert and this method of high-level feature extraction (HFE) substantiates the significance of our approach.

Here author [7] has presented a convex process for mutually learning feature weights and restrictions of SVM classifiers. Using support vector machine (SVM) classifiers for selecting appropriate features for significant for a selection of reasons such as simplification concert, computational competence, and feature understandability. Additionally, we related the proposed framework with $\mathrm{L}_{1}-\mathrm{SVMs}$ and provided and theoretical justification for its use as a feature selection method. Conventional SVM approaches to feature selection characteristically remove features and learn SVM parameters separately. Independently achieving these two steps strength consequence in a loss of information communicated to the classification process. This paper proposes a convex energybased structure to together complete feature selection and SVM constraint learning for linear and non-linear kernels. Experiments perform on seven standard datasets demonstrate significant reduction of features and different classification problems showed that our method produced SVM classifiers that used sparse sets of features and support vectors while maintaining classification performance.

In this paper [8] author is proposed to the use of discrete wavelet transform (DWT) both for signal preprocessing and indicate segments feature extraction as an unconventional to the frequently used discrete Fourier transform (DFT). Feature vectors belonging to take apart signal segments are then classified by a viable neural network as one of techniques of cluster analysis and processing. The paper provides a comparison of classification effects using different techniques of feature extraction most suitable for EEG signal components detection. Difficulties of multichannel segmentation are talk about in this association also. The outcomes of this method of signal classification can be more try to get better by a variety of techniques but one the most important difficulties is in the accurate definition of signal segment features using both its frequency-domain and time-domain possessions and it give the impressions that the DWT can be used in this area very proficiently. Additional studies will be also dedicated to the use of principal component analysis to come across essential signal components from all scrutinized channels for the tracking global segmentation.

In this research author [9] has try to get involves the Iris Localization based on morphological or set theory which is well in shape detection. This algorithm implemented morphological operation for the inner and outer iris edge detecting and positioning and has the advantages of short localization time and high localization precision. Principal Component Analysis (PCA) is used for preprocessing, in which the elimination of superfluous and unnecessary data is completed. Applications such as Median Filtering and Adaptive thresholding are used for managing the differences in lighting and noise. Features are takeout using Wavelet Packet Transform (WPT). As a final point matching is completed using KNN. Experimental results of the proposed method are better than the prior method and are proved by the consequences of different parameters. This method signify that the proposed algorithm is a kind of efficient iris locating algorithm in the characteristic of time inspired and localization precision.

\section{PROPOSED METHODOLOGY}

1. Take an input image.

2. Apply genetic algorithm based SIFT algorithm for the extraction of features from the image.

\section{Basic steps of GA}

1. Start

2. $\mathrm{T} 1=0$

3. Initialize the population of genetic $\mathrm{p} 1(\mathrm{~T} 1)$

4. Now Compute and calculate the fitness value p1(T1)

5. $\mathrm{T} 1=\mathrm{T} 1+1$

6. Check if the termination criterion satisfies

7. If yes then move and achieved go to step 10

8. Now select $\mathrm{p} 1(\mathrm{~T} 1)$ from $\mathrm{p} 1(\mathrm{~T} 1-1)$

9. crossover both the populations $\mathrm{p}(\mathrm{T} 1)$

10. and Mutate these populations $\mathrm{p}(\mathrm{T} 1)$

11. Now go to step 3

12. output the best population and stop

13. End

\section{Selection \& Cross Over}

Both the selection and crossover of the real-coded GA have been used to insure a steady convergent behaviour of the GA. The trade off we had to make is the well known trade-off between exploration and exploitation present in any search method including GA. The convergent exploitation assured by selection and crossover should well-balance the wide exploration effect achieved by our mutation operator. The selection method was chosen as a combination between binary 
tournament which has a constant and relatively high selection pressure (Miller 1996), with a $K$ - elitist scheme (Bäck 1991) that assures the preservation of the $K$ best individuals in the population.

Fitness value of each pixel can be calculated by taking the sum of average of all the intensity pixels of the block region. Fitness function in genetic algorithm is used to check the fitness of the pixel means in genetic algorithm when child chromosomes can be generated then the value of child is greater than the fitness value otherwise these child chromosomes are rejected.

\section{Mutation}

Mutation operator has been chosen to insure high levels of diversity in the population. We introduced PCA-mutation in (Munteanu 1999b), and shown that it has very good capabilities in maintaining higher levels of diversity in the population. We briefly summarize the PCA-mutation operator, as follows: The population $\mathbf{X}$ of the GA can be viewed as a cloud of $N$ points in a $l$-dimensional space, where $N$ is the size of the population and $l$ is the length of the chromosome.

parents

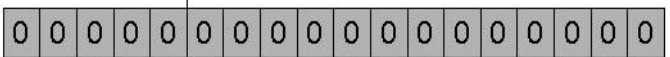$$
\begin{array}{|l|l|l|l|l|l|l|l|l|l|l|l|l|l|l|l|l|l|}
\hline 1 & 1 & 1 & 1 & 1 & 1 & 1 & 1 & 1 & 1 & 1 & 1 & 1 & 1 & 1 & 1 & 1 & 1 \\
\hline
\end{array}
$$

children

$$
\begin{array}{|l|l|l|l|l|l|l|l|l|l|l|l|l|l|l|l|l|l|}
\hline 0 & 0 & 0 & 0 & 0 & 1 & 1 & 1 & 1 & 1 & 1 & 1 & 1 & 1 & 1 & 1 & 1 & 1 \\
\hline
\end{array}
$$

\section{\begin{tabular}{|l|l|l|l|l|l|l|l|l|l|l|l|l|l|l|l|l|l|}
\hline 1 & 1 & 1 & 1 & 1 & 0 & 0 & 0 & 0 & 0 & 0 & 0 & 0 & 0 & 0 & 0 & 0 & 0 \\
\hline
\end{tabular}}

a. Alter each gene independently with a probability $p_{m}$

b. $\quad p_{m}$ is called the mutation rate

Typically between 1/population size and 1/ chromosome_length.

parent

$$
\begin{array}{|l|l|l|l|l|l|l|l|l|l|l|l|l|l|l|l|l|l|}
\hline 1 & 1 & 1 & 1 & 1 & 1 & 1 & 1 & 1 & 1 & 1 & 1 & 1 & 1 & 1 & 1 & 1 & 1 \\
\hline
\end{array}
$$

child

\begin{tabular}{|l|l|l|l|l|l|l|l|l|l|l|l|l|l|l|l|l|l|}
\hline 0 & 1 & 0 & 0 & 1 & 0 & 1 & 1 & 0 & 0 & 0 & 1 & 0 & 1 & 1 & 0 & 0 & 1 \\
\hline
\end{tabular}

\section{SIFT FEATURE EXTRACTION}

1. Find the points, whose surrounding patches (with some scale) are distinctive

2. An approximation to the scale-normalized Laplacian of Gaussian

$$
\begin{gathered}
L(x, y, \sigma)=G(x, y, \sigma) * I(x, y) \\
G(x, y, \sigma)=\frac{1}{2 \pi \sigma^{2}} e^{-\left(x^{2}+y^{2}\right) / 2 \sigma^{2}} \\
D(x, y, \sigma)=(G(x, y, k \sigma)-G(x, y, \sigma)) * I(x, y) \\
=L(x, y, k \sigma)-L(x, y, \sigma) .
\end{gathered}
$$

4. RESULT ANALYSIS

Table 1. Comparison of Computational Time

\begin{tabular}{|l|l|l|l|}
\hline S. No. & Image & Existing Work & Proposed Work \\
\hline 1 & lena.jpg & 21.142608 & 4.397908 \\
\hline 2 & lena1.jpg & 5.02584 & 1.33365 \\
\hline 3 & baboon.bmp & 5.94428 & 1.611818 \\
\hline 4 & peppers256.jpg & 5.133445 & 1.252713 \\
\hline 5 & testfi.jpg & 5.760211 & 1.079809 \\
\hline 6 & testfile.jpg & 189.835558 & 121.343884 \\
\hline
\end{tabular}

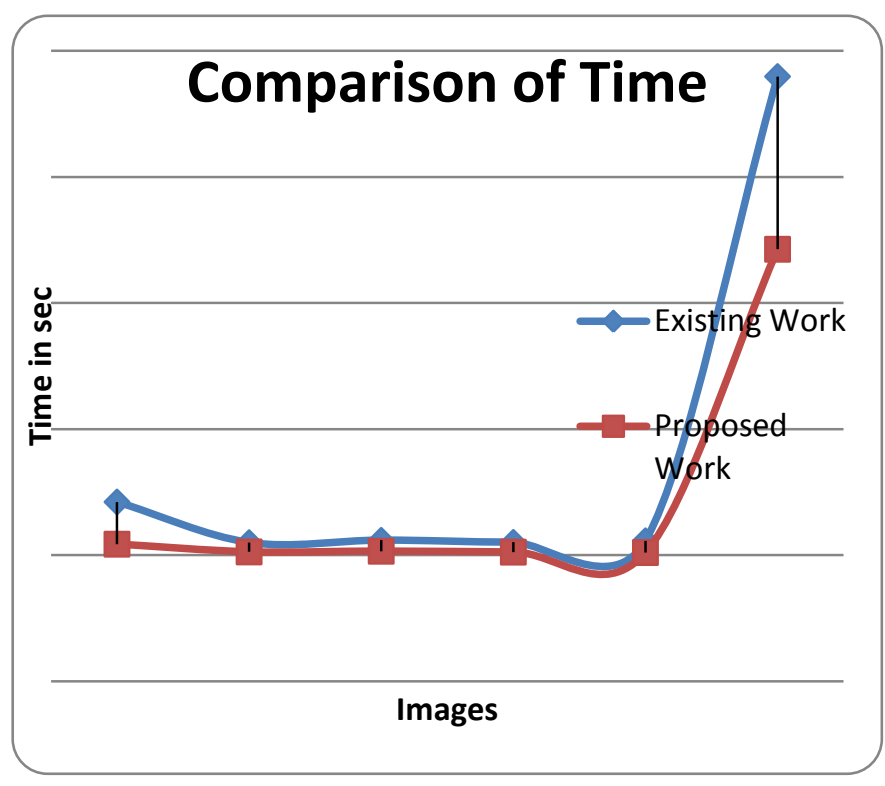

Figure 2. Comparison of Computational Time

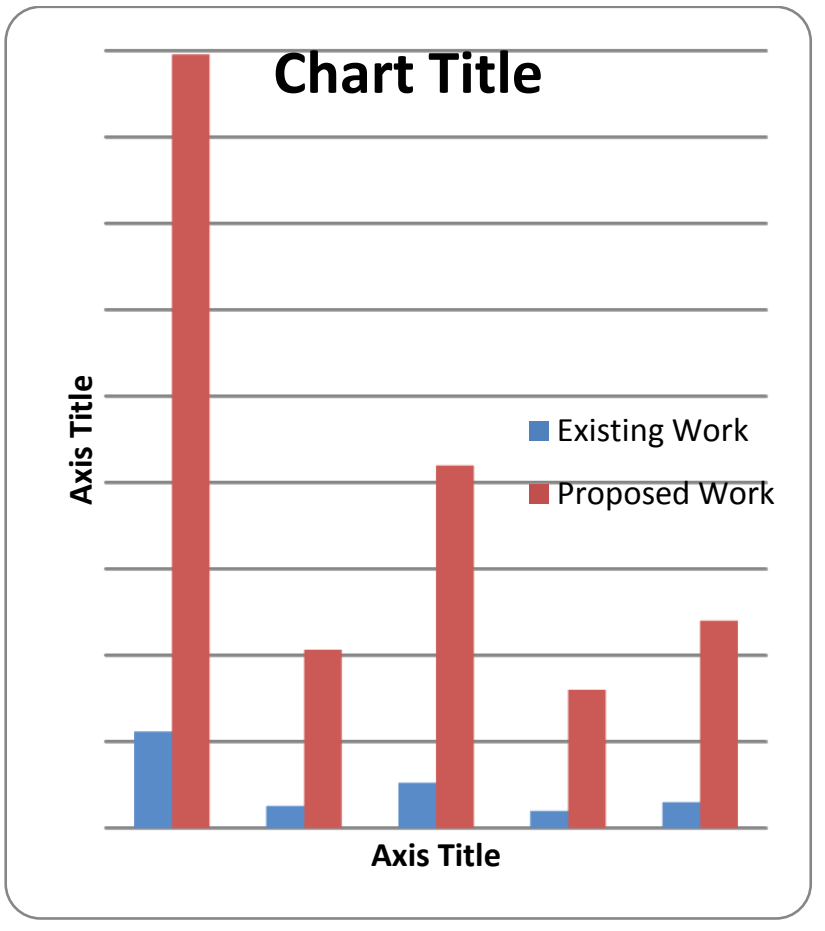

Figure 3. Comparison of Features Extracted 
Table 2. Comparison of Features Extracted

\begin{tabular}{|c|c|c|c|}
\hline S. No. & Image & Existing Work & Proposed Work \\
\hline 1 & lena.jpg & 2240 & 17920 \\
\hline 2 & lena1.jpg & 516 & 4128 \\
\hline 3 & baboon.jpg & 1050 & 8400 \\
\hline 4 & peppers256.jpg & 400 & 3200 \\
\hline 5 & testfi.jpg & 600 & 4800 \\
\hline 6 & testfile.jpg & 19528 & 156224 \\
\hline
\end{tabular}

\section{CONCLUSION}

The methodology implemented here for the extraction of features using combinatorial method of applying genetic algorithm on SIFT (Scale Invariant Feature Transformation) improves the number of features as compared to other existing techniques implemented for the feature extraction. The experimental results and analysis shows the performance of the proposed methodology.

\section{REFERENCES}

[1] N. C. Yang, W. H. Chang, C. M. Kuo, et al., "A fast MPEG-7 dominant colour extraction with new similarity measure for image retrieval", Journal of Visual Comm. and Image Retrieval, vol. 19, (2008), pp. 92-105.
[2] M. M. Islam, D. Zhang and G. Lu. "A geometric method to compute directionality features for texture images", In Proc. ICME, (2008), pp. 1521-1524.

[3] S. Arivazhagan and L. Ganesan, "Texture classification using wavelet transform", Pattern Recognition Letters, vol. 24, (2003), pp. 1513-1521.

[4] H.J.Baeand S.H.Jung, Image Retrieval Using Texture Based on DCT,Proc. Of the international conference on Information and communications security, vol.2pp.10651068,1997

[5] Erkan Bostanci, NadiaKanwal, Adrian F. Clark, "Spatial Statistics of Image Features for Performance Comparison" IEEE TRANSACTIONS ON IMAGE PROCESSING, VOL. 23, NO. 1, JANUARY 2014.

[6] Jean-Philippe Vert, Tomoko Matsui, "High-Level Feature Extraction Using Svm With Walk-Based Graph Kernel, 2007.

[7] Minh Hoai Nguyen, Fernando de la Torre, "Optimal feature selection for support vector machines" Pattern Recognition 43, 584-591, 2010.

[8] Ales Prochazka and Jaromır Kukal, "Wavelet Transform Use for Feature Extraction and EEG Signal Segments Classification.

[9] Suganthy, M. and P. Ramamoorthy, "Principal Component Analysis Based Feature Extraction, Morphological Edge Detection and Localization for Fast Iris Recognition", Journal of Computer Science 8 (9): 1428-1433, 2012 ISSN 1549-3636. 